\title{
Mental-rotation effect: a function of elementary stimulus discriminability?
}

\author{
Bettina Förster ף, Ralf-Peter Gebhardt, Karen Lindlar, Martina Siemann, Juan D Delius\# \\ Allgemeine Psychologie, Universität Konstanz, D-78434 Konstanz, Germany \\ Received 9 May 1996
}

\begin{abstract}
It is well known that when humans have to decide whether two differently oriented shapes are identical or mirror images their performance deteriorates as a function of the orientation disparity (mental-rotation effect). Here it is shown that the effect can also be obtained reliably with non-mirror-image, arbitrarily different polygons provided they are previously selected to be hard to discriminate. The slope of the decision speed versus orientation disparity functions was found to be inversely related to the discriminability of shapes under conditions of no, ie $0^{\circ}$, orientation disparity. Easily discriminable polygon pairs yielded essentially flat, no-effect functions. The arbitrary polygons that were more difficult to discriminate produced a rotation effect that was similar to those of mirror-image polygon pairs. Mirror images in this context may only be a special case of hard-to-discriminate stimuli. We also show that the speed of judging whether simple lines were of the same or different length was similarly subject to a rotation effect provided that the length differences were sufficiently small, ie when their baseline dicriminability was poor enough. It is suggested that the mental rotation of complex shapes (eg polygons) may build on rotation effects pertaining to the simpler elements of which they are composed. Further, some special effects associated with the rotation of such simpler components may explain certain peculiarities apparent in orientation invariance functions obtained with complex stimuli.
\end{abstract}

\section{Introduction}

Shepard and Metzler (1971) discovered that, when human subjects had to decide whether a comparison shape was the same as or different from a standard shape, the latencies (and the errors!) rose as functions of the angular-orientation disparity between the two shapes. They suggested that this effect reflected the operation of a timeconsuming and error-prone process which they called mental rotation. Indeed, subjects participating in such experiments often report that they gradually turn the image of one of the shapes in their minds until the orientation disparity is eventually compensated for and a template-matching-type comparison becomes possible. Much research ensued on the rotation effect, as we shall neutrally call the empirical phenomenon, most of it motivated by a controversy over whether it is truly based on an analogical, whole-image operation or rather on a propositional processing of feature lists (Kosslyn et al 1979; Pylyshyn 1981). Although that particular dispute, to which we return later, still remains largely unresolved, the ensuing research revealed, among other things, that the rotation effect is by no means a universal phenomenon in tasks demanding visual-orientation-invariance competences.

It is true that many later investigators who, as Shepard and Metzler had done, used mirror-image shapes as odd comparison stimuli have invariably replicated the rotation effect (reviews in Corballis 1988; Shepard and Cooper 1982). However, nearly all the investigators who used non-mirror-image odd shapes failed to find a rotation effect, finding instead that reaction times and/or error rates were not affected in any particular way by mounting angular disparities between standard and comparison stimuli (eg Aulhorn 1948; Corballis and Nagourney, 1978; Corballis et al 1978; Delius and Hollard 1995; Eley 1983; Foster and Mason 1979; Pierret and Peronnet 1994; Simion et al 1982; Tilgner and Hauske 1980; White 1980; Zimmer and Fröhlich 1991).

I Present address: Department of Psychology, University of Auckland, Private Bag, New Zealand. \# Author to whom requests for reprints should be addressed. 
A result of Takano (1989) is particularly revealing in this respect. He used odd shapes that differed with respect to both a mirror-image feature and a non-mirror-image feature. As long as subjects were not aware of the latter feature they showed a rotation effect but as soon as they became aware of it, they produced a flat performance function.

Only a few researchers using purely non-mirror-image odd shapes have reported rotation-like effects (Folk and Luce 1987; Jolicoeur and Landau 1984; Larsen 1985; Pylyshyn 1979; Tarr and Pinker 1989; Valentine and Bruce 1988). As we will describe later, in section 5 , these researchers either did not attempt to identify or did not satisfactorily manage to identify the cause of their unusual results although it is suggestive that they all employed comparatively special stimuli and/or methods. Not surprisingly the prevalent opinion remains that a reliable and definite rotation effect can only be obtained with mirror-image shape pairs (reviewed in Corballis 1988). This is thought to be so because the orientation uncertainty that is intrinsic to mirrored shapes (Ittelson et al 1991; see also Turnbull and McCarthy 1996) is potentiated by the additional shape rotations.

We became concerned with this issue after finding that pigeons did not show a rotation effect when tested with mirror-image shapes [Delius and Hollard 1987; Hollard and Delius 1982; compare Vauclair et al (1993) for partially equivalent findings in baboon monkeys]. Humans tested with the same procedures as the pigeons yielded the expected rotation effect (Delius and Hollard 1995). Some older data suggested that pigeons, possibly because of a specialisation of the avian visual system, might find mirror-image shape pairs comparatively easier to distinguish than humans do (Corballis and Beale 1976). Humans are well known to have difficulties with differentiating mirror-images, a much-cited example being the trouble that school beginners have with distinguishing the printed letters b, d, p, and $\mathrm{q}$ (cf Kolers and Perkins 1969). The hypothesis that pigeons might not show a rotation effect because they find mirror images relatively more easy to discriminate than humans do, was partially supported by subsequent results (Delius and Hollard, 1995; Lohmann et al 1988).

This circumstance led us to consider whether the variations in the incidence and, indeed, magnitude of rotation effects found in humans could be due to the fact that the stimuli employed simply differed in basic discriminability. With basic, or baseline, differences in stimulus discriminability we refer to the established fact that even in the absence of any orientation disparities, the accuracy and/or speed with which subjects decide whether two visual shapes are different or identical tends to vary (within certain limits) as a function of the degree of physical dissimilarity between the odd stimuli. Would rotation effects come to the fore with non-mirror-image odd shapes if these were designed and preselected to be particularly hard to discriminate? Moreover, would the magnitude of the rotation effect increase as the basic discriminability of such shape pairs diminished? Would the magnitude of the rotation effects obtained with hard-todiscriminate arbitrary stimuli perhaps be comparable in magnitude to those obtained with mirror-image shapes? Finally, would much simpler shapes than those traditionally used also yield a rotation effect if they were sufficiently difficult to discriminate?

To answer the first questions we performed an experiment with the same kind of random polygons used in previous rotation-effect research (Cooper, 1975). However, such polygons have a disadvantage in that it is difficult to specify which physical features ultimately make them easy or hard to discriminate. To answer the latter question we carried out two experiments with much simpler shapes. These were straight lines where the comparison stimuli were either identical to a standard stimulus or differed in length from it by a specified smaller or larger amount (ie in basic discriminability). Any differences in rotation effect that we might subsequently establish would thus be directly traceable to objective stimulus properties. 
A demonstration of a rotation effect with such elementary stimuli would also be indirectly informative on the analogical versus propositional controversy alluded to earlier. This issue has been recently revived in a modified guise through the proposal of a neural network model of visual pattern rotation (Goebel, 1990; see also Morgan 1983). In the model it is proposed that the orientation invariance of complex stimuli is achieved through a piecemeal rotation of the line components and not through a holistic rotation of their analogue images as suggested by other formal, albeit physiologically unrealistic, models (Messner and Szu 1985; Reitboek and Altman 1984; Sheng 1989). An actual demonstration of a rotation effect with simple line stimuli would clearly add some psychological credence to the network proposal that already has received some, though indirect, neurophysiological support (Georgopoulos et al 1989; Pellizer and Georgopoulos 1993).

\section{Experiment 1}

Random polygons as used in several classical mental-rotation studies (Shepard and Cooper 1982) were employed as stimuli. First, non-mirror-image pairs of random polygons were designed and selected to differ in operational discriminability when presented with $0^{\circ}$ orientation disparities. Mirror-image polygon pairs were then added and we then examined what effect orientation disparities between pair members had on the discrimination performance.

\subsection{Methods}

Pairs of non-mirror-image, twelve-sided random polygons (Attneave 1956), each with a surface area of $14 \pm 1 \mathrm{~cm}^{2}$ were drawn. The polygons composing six pairs differed in the extent of their radii by $\pm 15 \%$ at one of the corners, those composing six further pairs differed at four corners and those composing six final pairs differed at eight corners by the same amount. Care was taken that the polygons belonging to the various pairs were clearly distinct from each other. For a preselection these pairs were reproduced in a booklet, all in their original $0^{\circ}$ orientation and were rated for intrapair similarity/dissimilarity on a 5-point scale by twenty observers. Three sets of four nonmirror-image pairs, those most consistently rated with respectively 1,3 , and 5 points, were retained. The set was then completed with four unselected, additional pairs, each composed of a random polygon and its vertically reflected mirror image.

2.1.I Discriminability. The sixteen stimulus pairs listed above were transferred to the graphics file of a personal computer. The pairs were then converted to triplets, with the standard polygon displayed in the middle, an identical comparison polygon displayed to the right or left side, and the modified comparison polygon displayed to the alternative side, all in their original $0^{\circ}$ orientation. Each triplet existed in a right and left odd-stimulus version. On the monitor the polygons appeared as white shapes of about $3 \mathrm{~cm}$ diameter (about $2.5 \mathrm{deg}$ visual angle), centres $8 \mathrm{~cm}$ apart, on a dark background.

Sixteen students (eight male, eight female) between 19 and 27 years of age participated. They were positioned with their eyes about $70 \mathrm{~cm}$ from the monitor and were instructed to react as accurately and as fast as possible. The subjects initiated the trials by pressing the centre, down-arrow key on the keyboard and then indicated which of the flanking stimuli was identical to the standard stimulus by pressing either the leftarrow or the right-arrow key. Note that the triple-stimulus arrangement prevented the incidence of separate same-pair and odd-pair trials. Correct or incorrect choices were signalled with the German words for 'fine!' or 'poor!' appearing on the monitor. Each of the 32 polygon triplets was shown twice, all in quasi-random order (Gellermann 1933) during a single session that lasted about $40 \mathrm{~min}$. 
2.1.2 Rotation. A new group of twenty-four students (twelve female, twelve male) aged between 18 and 32 years participated. The apparatus and general procedure were the same as described above. However, the polygon stimuli employed were those selected as a result of the previous phase (figure 1). Within each of the constellations selected the standard (middle) polygons were tilted from their original orientation by either $0^{\circ}$, $30^{\circ}, 60^{\circ}$, or $120^{\circ}$ clockwise in different triplet versions. This was done to prevent any by-rote memorisation strategy. The same and odd comparison polygons of a given triplet were shown identically rotated with respect to the sample stimulus by either $0^{\circ}$, $60^{\circ}, 120^{\circ}$, or $180^{\circ}$ clockwise. The single session involved the presentation of 14 randomly selected warm-up triplets followed by the presentation, in random sequences, of all the 256 polygon triplets arising from the above arrangements. Subjects took about 40 min to complete the session.

easy

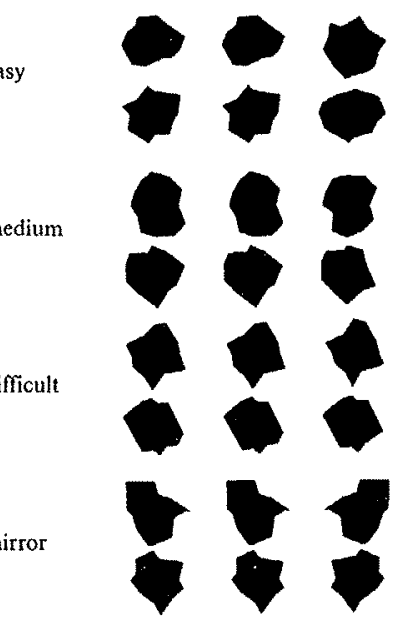

Figure 1. Examples of stimulus displays employed in experiment 1 . Only versions with $0^{\circ}$ tilt, $0^{\circ}$ disparity, and odd stimulus to the right are shown.

\subsection{Results and discussion}

2.2.1 Discriminability. The results were assessed by examining the mean error rates and the mean reaction times for all polygon triplet types. The overall error rate amounted to $7.7 \%$ and varied little. The two non-mirror-image polygon triplet types that yielded respectively the shortest, typically intermediate, and longest average reaction times were retained for the categories easy, medium, and difficult to discriminate. The average latencies associated with each of these non-mirror-image polygon categories (1185 ms, $1714 \mathrm{~ms}$, and $2673 \mathrm{~ms}$ ) were significantly different [Page test: $L_{3,10}=137$, $p<0.001$ (Siegel and Castellan 1988)]. Two mirror-image polygon pairs were selected on the basis of having yielded the most-similar average reaction times. Their common mean was $1551 \mathrm{~ms}$.

2.2.2 Rotation. The mean reaction times obtained were strongly influenced by the stimulus categories $\left(F_{3,69}=72.3, p<0.0001\right)$ and the orientation disparities $\left(F_{3,69}=63.9\right.$, $p<0.0001)$. However, most important was the significant disparity and category interaction $\left(F_{9,207}=16.5, p<0.0001\right)$. It indicated that the rotation effects obtained were markedly modulated by the categories variable.

Figure 2 presents the mean reaction times and error rates as functions of orientation disparity and discrimination category. The speed functions pertaining to the mirror-image 
polygon triplets and the difficult non-mirror-image polygon triplets reveal a similar rotation effect while the functions corresponding to the medium and easy triplets are increasingly flatter. Even though the analysis of the error rates yielded no significant effects, the functions associated with them exhibit weak trends but similar to those of the latencies. A valid bivariate analysis of variance of error rates and reaction times is unfortunately precluded by the discrete and continuous nature of, respectively, the error and the time variables. This remark, incidentally, also applies to the two experiments that follow.

A regression analysis on the reaction-time data yielded the intercepts and slopes of the linear components (all significant to at least the $p<0.05$ level) listed in table 1 . The overall mean error rates are also listed in the table. Errors (indexing the overall task difficulty) and intercepts (indexing the reaction times at $0^{\circ}$ disparity) correlated positively with the slopes (indexing the rotation-effect magnitudes; $r_{s, 8}=0.86$ and $r_{s, 8}=0.82$, respectively; $p<0.05$ in each case). The latter correlation accords with the hypothesis that the baseline discriminability of a stimulus pair at $0^{\circ}$ disparity determines, at least in part, the size of the rotation effects that it generates.

Table 1. Experiment 1: Percentage errors, latency intercepts (in ms) and slopes (in $\mathrm{ms} /{ }^{\circ}$ ) corresponding to the different polygon variants (with ranks in brackets).

\begin{tabular}{lrrr}
\hline Variant & Error & Intercept & \multicolumn{1}{l}{ Slope } \\
\hline easy I & $1.3(2)$ & $1053(1)$ & $2.9(3)$ \\
easy II & $0.3(1)$ & $1212(2)$ & $1.0(1)$ \\
medium I & $3.1(3)$ & $1523(3)$ & $1.9(2)$ \\
medium II & $4.5(4)$ & $1931(4)$ & $7.3(4)$ \\
difficult I & $13.8(5)$ & $3418(7)$ & $10.2(5)$ \\
difficult II & $24.5(7)$ & $3851(8)$ & $13.0(7)$ \\
mirror I & $21.6(6)$ & $2937(5)$ & $12.1(6)$ \\
mirror II & $25.7(8)$ & $2884(6)$ & $18.1(8)$ \\
\hline
\end{tabular}

The reaction times are somewhat longer than in studies involving only mirrorimage polygons (Shepard and Cooper, 1982; cf Folk and Luce 1987). However, in contrast to the prevalent but questionable practice (cf Ulrich and Miller, 1994), our latency data were not censored in any way. Nevertheless, the main cause for the relative slowness is likely to have been the complexity of our task, which involved the presentation of a large number of different stimuli. The fact that even the straightforward $0^{\circ}$, no-disparity condition and also the conventional mirror-image stimuli yielded comparatively long latencies supports this view. It is also true that quadratic trends affected most of our latency functions (figure 2). Although the linearity of the rotation effect has been commonly stressed, nonlinearities have been reported quite often (eg Hock and Tromley 1978; Jolicoeur 1985; Koriat and Norman 1985; Young et al 1980). Removal of error trials from our data did not reduce the convex trends, indicating that short-latency, default choices were not involved. More-linear functions might have emerged if the subjects had been given more practice. Increased rotation experience with given stimuli seems to enhance a concave trend (Koriat and Norman 1985; Young et al 1980). In any case, the simultaneous same-different procedure we employed cannot be made responsible for the long latencies and the nonlinearities. Delius and Hollard (1995), using a simpler stimulus set than here, obtained a fast and linear effect with it. Also, another experiment of ours (Siemann, in preparation), closely equivalent to the present one but involving a conventional successive same-different method, produced results that were quite similar to the present ones. 

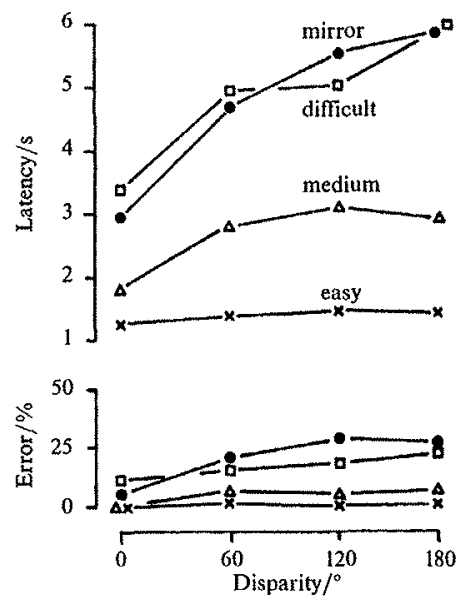

Figure 2. Experiment 1, rotation phase: mean error rates and reaction times plotted as a function of orientation disparity, with discrimination category as a parameter.

Regardless of these details, the present results support the hypothesis that the basic stimulus discriminability is an important factor in determining the presence and magnitude of the rotation effect. Non-mirror-image polygon pairs selected to be difficult to distinguish at $0^{\circ}$ disparity yield choice latencies that rise with increasing orientation disparities, much as those of mirror-image polygon pairs generally do. The size of the rotation effect occurring with these polygons compared advantageously with that obtained with the mirror-image polygons. There is nothing to suggest that the effects attaching to the two kinds of stimuli were due to different mechanisms. Indeed, most of our subjects, when questioned afterwards, were clearly not aware of having dealt with different types of stimuli.

\section{Experiment 2}

In experiment 1 it was shown that basically difficult-to-discriminate, non-mirror-image polygons can yield rotation effects comparable to those of the classically employed mirror-image polygons. However, as can be seen from the first six rows of figure 1 , it is not easy to say which precise features are responsible for the differences in discriminability. In the present experiment we thus explored whether component elements of such polygons, straight lines of differing length, would by themselves yield a rotation effect. We employed triplets assembled from a sample line of standard length, an identical comparison line, and an odd comparison line differing in length by a specified amount. Note that with lines (as with all axially elongated and orthogonally symmetric shapes) nonredundant rotations on the plane could only amount to less than $180^{\circ}$, instead of the usual full circle.

\subsection{Methods}

The same apparatus and general procedures as in experiment 1 were employed. However, line stimuli were used instead of polygons. A chin support located $120 \mathrm{~cm}$ from the monitor ensured standardised viewing.

3.1.1 Discriminability. Ten students (six female, four male) between 20 and 31 years old participated. Each trial involved the simultaneous side-by-side presentation of three white lines about $1.5 \mathrm{~mm}$ wide. They were all either vertically or horizontally oriented (tilt variable), midpoints $7.5 \mathrm{~cm}$ apart. The middle standard line was always $5 \mathrm{~cm}$ 
long (2.38 deg visual angle). Quasi-randomly, either the right or the left comparison line was of equal length. The other, odd comparison line was either $4.2,4.5,5.5$, or $5.8 \mathrm{~cm}$ long (figure 3). The length differences, $0.5 \mathrm{~cm}$ [Weber fraction (difference in length/length) $=0.10$, difficult category] and $0.8 \mathrm{~cm}$ (Weber fraction $=0.16$, easy category), selected were markedly larger than the just-noticeable differences psychophysically determined by Ono (1967) with highly trained observers. Preliminary trials had shown that Ono's threshold differences could not sustain a reliable line-length discrimination by inexperienced subjects.

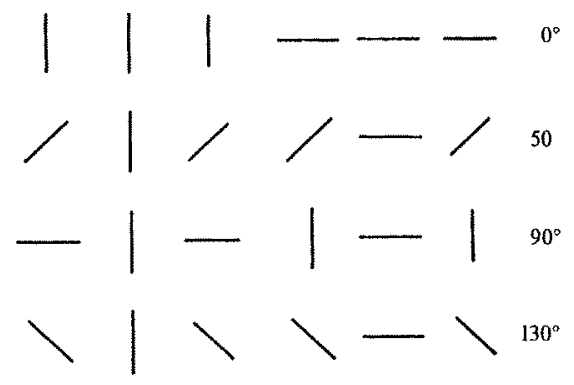

Figure 3. Examples of stimulus displays used in experiment 2, approximately to scale: standard with vertical (left) and horizontal tilt (right). Comparisons are shown with $0^{\circ}, 50^{\circ}, 90^{\circ}$, and $130^{\circ}$ orientation disparities. Only shorter odd comparison lines are shown, to the right.

Pressing the down-arrow key caused a stimulus triplet to appear and subjects were expected to press the left-arrow or the right-arrow key corresponding to same comparison line. Feedback about correct and incorrect choices was given with the words 'fine!' or 'poor!' replacing the stimuli, the latter word accompanied by a $2 \mathrm{~s}$ buzz. First there were 3 randomly chosen stimulus triplets with the correct choices marked on the screen, then 5 similarly selected warm-up trials and then 128 proper trials involving 8 presentations of each of all the 16 triplet types, in randomised order.

3.1.2 Rotation. A new group of eighteen students (ten female, eight male) aged between 20 and 31 years participated. The apparatus and procedure were the same as just described. The stimuli validated during the previous phase were used, but now both comparison stimuli deviated in orientation from that of the standard stimulus by either $0^{\circ}, 38^{\circ}, 50^{\circ}, 63^{\circ}, 90^{\circ}, 117^{\circ}, 130^{\circ}$, or $142^{\circ}$ clockwise. The particular oblique orientations were chosen because they yielded reasonably smooth and straight lines on the pixel raster of the monitor. All lines were rotated about their midpoint on the display plane. The single session began with the presentation of 3 randomly chosen stimulus triplets with the correct choices marked, followed by 14 randomly selected warm-up unmarked trials, and was completed with 256 proper trials according to a balanced, randomisedorder design.

\subsection{Results and discussion}

3.2.1 Discriminability. During the no-disparity phase the triplets with the small and large length difference yielded mean choice latencies of $1162 \mathrm{~ms}$ and $1412 \mathrm{~ms}$ and mean error rates of $3.1 \%$ and $11.4 \%$, respectively. Both differences were significant (Wilcoxon tests: $T_{10}=55, p<0.001$; and $T_{10}=54, p<0.01$, respectively).

3.2.2 Rotation. The mean reaction times were significantly influenced by the orientationdisparity factor $\left(F_{7,119}=6.5, p<0.001\right)$, indicating the presence of a rotation effect. Figure 4 presents a summary of the overall results. Mean reaction times and error rates are shown as a function of orientation disparity regardless of discrimination category. 

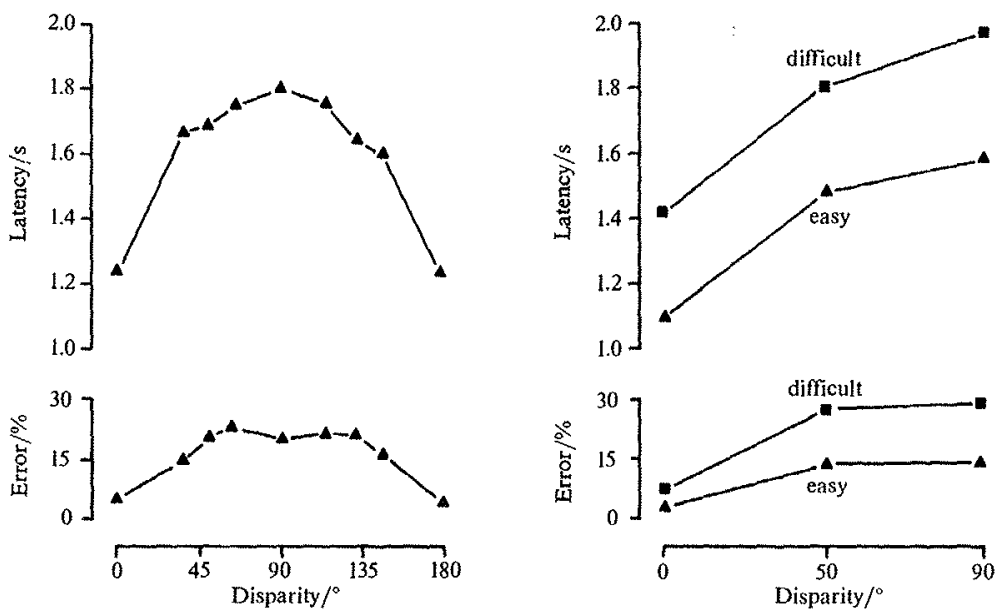

Figure 4. Experiment 2, rotation phase: mean reaction times and error rates as a function of orientation disparities.

Figure 5. Experiment 2, rotation phase: mean reaction times and percentage errors as a function of orientation disparity, with discriminability as a parameter. For conciseness, the data belonging to the various oblique disparities are shown averaged and plotted at $50^{\circ}$.

Both functions rise towards a maximum at $90^{\circ}$ orientation disparity reproducing the usual rotation effect, although compressed within $180^{\circ}$ as explained earlier. The symmetry of rotation functions is explained by assuming that subjects can compensate orientation disparities both clockwise and anticlockwise in a closely equivalent manner.

Figure 5 shows the mean reaction times and error rates as a function of orientation disparity with discriminability categories as a parameter. In accordance with the significant category effect $\left(F_{1,17}=32.2, p<0.0001\right)$ and the absence of a significant disparity and category interaction there is an obvious difference in intercepts (basic discriminability) but not in slopes (rotation-effect magnitudes) between the two latency functions. The slopes of the linear components of the latencies versus disparity functions (6.5 and $6.7 \mathrm{~ms} /{ }^{\circ}$ ), although both significant at the $p<0.05$ level, were rather shallow compared with some of those obtained in the previous polygon experiments. This may well be due to the relative speed-for-errors trade-off noticeable in this experiment, reflected in the fact that in this instance the error rates also evinced a significant rotation effect (see below). As in the previous experiments, convex quadratic trends were superimposed on the linear trends and, again, these trends did not disappear when error trials were excluded.

Matters were slightly complicated because the latencies were affected by a significant interaction of disparity and tilt $\left(F_{7,119}=9.8, p<0.001\right)$. A split of the data according to the tilt of the standard lines revealed that the vertical-tilt condition yielded a steeper rotation effect than the horizontal-tilt condition. This probably was so because the vertical tilt, $0^{\circ}$-disparity configuration allowed an easy side-by-side comparison of line length (l|I) whereas the horizontal tilt, $0^{\circ}$-disparity configuration necessitated a more difficult end-on-end line comparison of line length (-- ). Among the $90^{\circ}$-disparity configuration, probably for a similar reason, the vertical-tilt condition $(-1-)$ seems to have been somewhat more difficult than the horizontal tilt condition $(|-|)$. All the oblique-disparity configurations $(/-/, \backslash \mid \backslash$, etc) seem to have been approximately equivalent in this respect. 
Whereas in the polygor-rotation experiment the choice latencies were relatively long and the error rates vere comparatively low, in the present experiment this was reversed. Indeed, here the error rates were significantly affected both by the discriminability categories $\left(F_{1,17}=10.5, p<0.01\right)$ and by the orientation disparities $\left(F_{7,119}=2.8, p<0.01\right)$. However, the interaction between disparity and discriminability suggested by the diverging error-rate functions shown in figure 5 (slopes of the linear components: $0.13 \% /^{\circ}$ and $0.30 \% /^{\circ}$ ), did not quite reach significance. Regardless of this, insofar as lines of differing length are undoubtedly features of random polygons, the rotation effects observed with the former may have contributed to the effects obtained with the latter stimuli.

\section{Experiment 3}

Although it was shown that a rotation effect can arise during the discrimination of the length of simple lines, in experiment 2 we did not quite establish that the basic discriminability of the line stimuli influenced the magnitude of the rotation effect. It seemed possible that an insufficient difference in basic discriminability and the tilt effect described above might have obscured a modulation of the rotation effect by the discriminability category factor. In the present experiment accordingly we employed line stimuli differing more clearly in basic discriminability and the experiment was designed to compensate for the influence of tilt by the introduction of an additional alignment variable. Since the rotation effect obtained in experiment 2 had been obviously symmetric for equivalent clockwise and anticlockwise angular disparities, the orientation disparities employed in this experiment ranged only between $0^{\circ}$ and $90^{\circ}$.

\subsection{Methods}

Apparatus and procedure were analogous to those used in experiment 2 except that the graphics and monitor employed had a higher resolution. The line stimuli could hence be presented with an even definition at the usual intermediate orientations. Since the stimuli were of better graphic quality, the chin support could stand closer $(65 \mathrm{~cm})$ to the monitor than in experiment 2. The lines also differed in length from those used in that experiment.

4.1.1 Discriminability. Ten students (five female, five male) between 19 and 28 years old took part. A trial involved the presentation of three white lines about $1 \mathrm{~mm}$ wide. A standard line $4.1 \mathrm{~cm}$ long ( $3.6 \mathrm{deg}$ visual angle) was shown in the centre of the screen. The comparison stimuli were located either to the right and left (horizontal alignment) or below and above (vertical alignment), with their midpoints $5.8 \mathrm{~cm}$ away from the centre of the standard line. All three lines were either horizontal or vertical (tilt variable). The odd comparison lines were either $3 \mathrm{~mm}$ (Weber fraction $=0.08$, difficult category) or $9 \mathrm{~mm}$ (Weber fraction $=0.22$, easy category) longer or shorter than the standard. The other comparison line was identical to the standard line. The subjects initiated trials by pressing the centre key of numerical field with the right index finger. When the lines appeared, subjects were expected to press one of the four numerical keys that corresponded topographically (up, down, left, right) with the location of the odd comparison stimulus. The stimulus configurations used were the balanced factorial combination of two tilts, two alignments, four line lengths, and two alternative odd-stimulus locations. The subjects saw each of these configurations four times, all in randomised order. The session, which lasted about $15 \mathrm{~min}$, began with 3 trials with marked correct stimuli, followed by 8 warm-up trials, and was completed by 128 proper trials.

4.1.2 Rotation. A new group of twenty students (twelve female, eight male) between 23 and 31 years old participated in this phase. Now both comparison lines appeared additionally rotated by either $0^{\circ}, 30^{\circ}, 60^{\circ}$, or $90^{\circ}$ against the standard line (disparity factor; 
figure 6). The subjects saw each of the factorial configurations four times, all in randomised order. The session consisted of 6 examples with marked correct stimuli, 16 warm-up trials, and the 512 true trials detailed above. Altogether the session lasted about $50 \mathrm{~min}$.

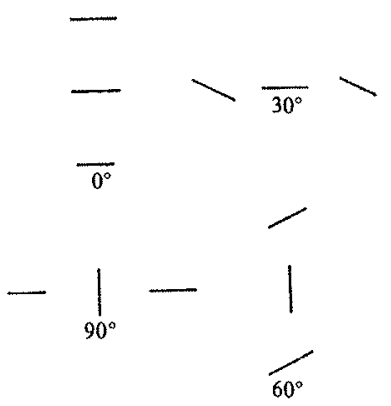

Figure 6. Examples of stimulus configurations employed in experiment 3, rotation phase. The vertical and horizontal stimulus alignments, the vertical and horizontal standard tilts, and the four comparison disparities are illustrated. Only shorter odd comparison configurations are shown.

\subsection{Results and discussion}

4.2.1 Discriminability. The first phase revealed that the easy and difficult categories differed significantly with respect to both error rates and reaction times $6.6 \%$ and $19.2 \%$ errors, reaction times $1320 \mathrm{~ms}$ and $1842 \mathrm{~ms}$, Wilcoxon tests, $T_{10}=55$ and $T_{10}=55$, respectively; $p<0.001$ in each case). The difference in basic line-length discriminability at $0^{\circ}$ orientation disparity was thus validatec.

4.2.2 Rotation. A summary of the results obtained during the main phase of the experiment is shown in figure 7. An analysis of variance of the latency data revealed that they were affected by a significant effect of disparity $\left(F_{3,57}=23.1, p<0.0001\right)$ and a significant effect of category $\left(F_{1,19}=53.7, p<0.0001\right)$. The interaction between these factors, however, did not reach significance, the slopes of the linear components
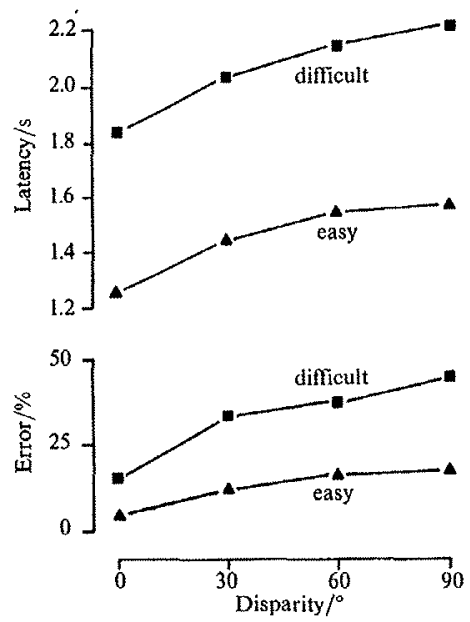

Figure 7. Experiment 3, rotation phase: mean reaction times and error rates as a function of the orientation disparities, with difficulty categories as a parameter. 
being too similar $\left(3.2\right.$ and $\left.3.8 \mathrm{~ms} /{ }^{\circ}\right)$. The error rates revealed a significant effect of disparity $\left(F_{3,57}=93.9, p<0.0001\right)$, a significant effect of category $\left(F_{1,19}=438.7\right.$, $p<0.0001$ ), and, importantly, also a significant interaction of category and disparity $\left(F_{3,57}=11.3, p<0.0001\right)$. That was the case in spite of the fact that the slopes of the corresponding linear trend components $\left(0.16 \% /{ }^{\circ}\right.$ and $\left.0.38 \% /^{\circ}\right)$ did not differ by much from those obtained in experiment 2. For uncertain reasons the residual error-rate variance was markedly lower in the present experiment.

The results demonstrate that the degree of discriminability of line length determined prior to any orientation disparity is related to the magnitude of the rotation effect obtained when disparities become operative. It is notable that the modulation of the rotation effect by the discriminability categories was principally reflected by the error data rather than the latency data. As in experiment 2 , subjects appeared biased towards a strategy of trade-off of variable errors for constant latencies.

Unlike in experiment 2, and because of the additional alignment factor, it is now possible to assemble the data according to properly equivalent stimulus configurations. Figure 8 shows, first, that for the condition with $0^{\circ}$ orientation disparity the mean reaction times and mean error rates (if difficulty categories are disregarded) corresponding to the parallel configurations are relatively better than those corresponding to the collinear configurations. Within each of these the horizontal alignnuents are again at some performance advantage over the vertical alignment. As already commented upon, the former result appears intuitively obvious but an account in terms of underlying mechanisms is nevertheless difficult to specify for either effect. It is true that it is known that lines falling on the upper field of vision are judged longer than those falling on the lower one (Masin and Agostini 1991), that lines falling on the left field of vision are judged longer than those falling on the right field (Bradshaw et al 1987; Milner et al 1992), and that vertical lines are judged longer than horizontal lines (Verillo and Irvin 1979). But none of these phenomena is likely to have been primarily involved in the above data variability since each of them should have been compensated for by the counterbalanced design. More specialised research on this point is needed.

It can also be seen from figure 8 that, regardless of these puzzling complications, an orientation disparity of the central sample line of $90^{\circ}$ against the comparison lines

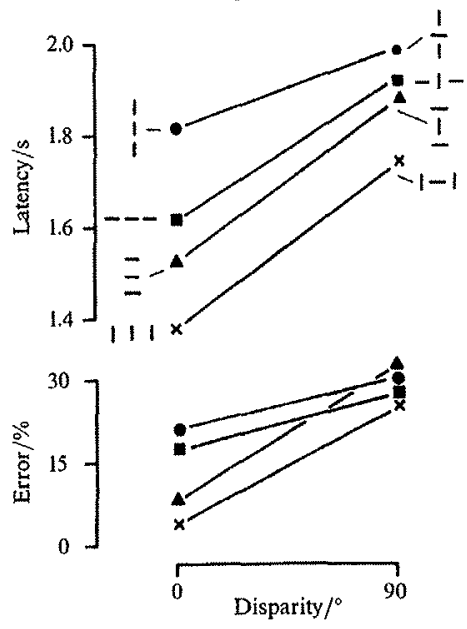

Figure 8. Experiment 3, rotation phase: mean reaction times and error rates (with difficulty categories disregarded) corresponding with configurations with $0^{\circ}$ and $90^{\circ}$ orientation disparities, sorted according to stimulus alignments and comparison tilts. 
for each of the constellations led to a fall in discrimination performance (increase in reaction times and error rates) relative to the corresponding $0^{\circ}$-disparity condition. This reaffirms that rotation effects consistently accompany difficult length discriminations of misoriented lines.

\section{General discussion}

With the experiments reported here we have established three facts. First, we have demonstrated that the incidence and magnitude of a rotation effect with non-mirrorimage, arbitrary shapes is determined by their basic discriminability. Second, we have shown that the rotation effect obtained with arbitrary shape pairs that are difficult to discriminate is commensurate with the effects obtained with the usual mirror-image shape pairs. Third, we have demonstrated that rotation effects occur not only with complex shapes but also with very elementary stimuli provided that they are relatively difficult to discriminate prior to any orientation disparities.

As already pointed out in section 1 the fact that rotation effects can sometimes occur with non-mirror-image shapes is not a novel finding. However, in the few extant studies reporting this fact, there is a failure to specifically attribute the rotation effect to the low discriminability of the stimuli used. Nevertheless, in retrospect, they all seem to have involved stimuli that were by themselves difficult to discriminate or, alternatively, that were embedded in tasks that made them difficult to discriminate, Pylyshyn (1979) found that a rotation effect could be obtained with rather special, only partially non-mirror-image odd shapes. Jolicoeur and Landau (1984) observed a subtle rotation effect with arbitrary odd shapes while using a tachistoscopic presentation followed by stimulus masking. Larsen (1985) reported that polygons that differed in both size and shape yielded an effect. Jolicoeur (1985) found that the time taken to name drawings of objects revealed a rotation effect. Folk and Luce (1987) first presented a standard polygon and then a mark indicating the projected disparity of a comparison polygon. The subjects called up the comparison polygon when they felt ready to decide whether it was the same or different. The preparation latencies revealed rotation effects and their variations were ascribed primarily to the complexity of the polygons and, secondarily, to their similarity. (1) Valentine and Bruce (1988) found rotation effects when pictures of not-too-different human faces were used as stimuli (see also Perret et al 1984). Last, Tarr and Pinker (1989) showed that arbitrary, though actually only slightly differing, shapes could yield a mental-rotation effect. In many further studies (see section 1) that involved non-mirror-image, arbitrarily different shapes researchers have of course reported a lack of rotation effects, arguably because the stimuli that they used were basically easy to discriminate.

That mirror-image shapes regularly yield a rotation effect is an undisputed fact. However, it has also been extensively documented that humans generally find mirrorimage patterns to be similar, and hard to distinguish (Bornstein et al 1978; Bradshaw et al 1976; Corballis and Beale 1976). That does not necessarily conflict with the argument that mirror images are particularly predestined to produce a rotation effect because they already differ intrinsically in a spatial respect (Corballis, 1988). It is worth pointing out, however, that the rotation effects obtained with mirror images can vary according to the shapes employed, probably because their basic discriminability is influenced by features other than their mirrored characteristic alone (Bethell-Fox and Shepard 1988; Larsen 1985; Petrusic et al 1978).

(1) A similar conclusion is sometimes erroneously attributed to Cooper and Podgorny (1976), who carried out a partially similar experiment. However, because of its particular design it did not really allow inferences about the role of complexity and similarity and, indeed, the authors did not strictly draw any such conclusions. 
The polygons used in experiment 1 can be viewed as closed arrangements of line-like edges. Indeed, that is how in the neuronal model of mental rotation mentioned earlier it is assumed they are actually perceived (Goebel 1990). In experiments 2 and 3 accordingly we explored whether rotation effects would emerge in connection with the discrimination of simple lines differing in length by small amounts. The results confirmed that expectation. It can be assumed that this relates to the fact that the assessment of small line-length differences per se, regardless of orientation, already involves a time-consuming, error-prone process (Hartley 1981; Ono 1967; Reed et al 1993; see also the discrimination phases of the above experiments). This process is presumably further complicated by orientation disparities. In experiment 3 we established that the magnitude of the rotation effect obtained was furthermore significantly influenced by the baseline discriminability of the lines. In this the rotation effects obtained with these simple stimuli did not differ from those associated with polygons (experiment 1). However, the variations in basic discriminability could now be referred to an unequivocal, objective characteristic, that of the length differences between the standard and the odd comparison stimuli.

These results with elementary stimuli allow one to entertain the hypothesis that the rotation effects obtained with random polygons could be due to a summation of partial rotation effects connected with elementary components of these complex shapes. Judgment processes akin to line-length comparisons seem to intervene, at least subjectively, when one identifies particular indentations or protrusions during the orientation-invariant recognition of polygons. Another process that seems similarly implicated is the identification of particular obtuse or acute corners. Even in the absence of actual experimental evidence, it seems obvious that, provided that there is a sufficient baseline difficulty, equality/inequality decisions about angle magnitudes are easier and faster with $\ll<\left(0^{\circ}\right.$ disparity) than they are with $\wedge<\wedge \quad\left(90^{\circ}\right.$ disparity $)$ or $><>\left(180^{\circ}\right.$ disparity) constellations. Accordingly, the rotation effect elicited by a given polygon pair should be strong if the pair involves hard-to-discriminate elementary features, or weak if the pair contains easy-to-discriminate elementary components. Mirror-image polygon pairs can be thought to yield particularly reliable rotation effects because they involve angle elements that require a basically difficult handedness discrimination $(J L L\lrcorner , L \neg, 7 L \Gamma)$. Of course, we realise that this listing of elementary shape components is by no means exhaustive: rotation effects can also arise with purely curvilinear shapes, for example.

Furthermore, given shapes may be composed of few or many elementary features (complexity). This is bound to affect baseline discriminabilities and thus rotation effects (cf Bethell-Fox and Shepard 1988; Folk and Luce 1987). Context factors such as the temporal and spatial structure of the task, the interference of concurrent discriminations, and the response demands associated with the task are likely to additionally modulate these phenomena.

Note that line elements yield rotation effects that peak at $90^{\circ}$ whereas angle elements can be expected to yield effects that peak at $180^{\circ}$ orientation disparities. If our conceptualisation is correct, then it predicts effects for polygons that are some combination of both kinds of functions, ie a function incorporating a linear component and a convex quadratic component within the $0^{\circ}$ to $180^{\circ}$ disparity range. This is precisely the kind of function we obtained in experiment 1 . But it is also true that other authors have reported quite linear functions (eg Shepard and Cooper 1982). One can speculate that such functions may arise when the convex quadratic component is compensated for by a concave quadratic component. Such a component is thought to arise when subjects become increasingly familiar with the standard orientation of the sample stimuli owing to a broadening of a stimulus generalisation gradient around that orientation (widened perceptual uprightness: Hock and Tromley, 1978; Young et al 1980). 
However, it has to be admitted that, as indicated by figures 5 and 7 , the functions concerning line elements already include a convex quadratic trend, suggesting a component function that peaks at $45^{\circ}$. At present we are inclined to attribute this to the well-known oblique effect, ie the fact that oblique lines are perceptually less defined than either vertical or horizontal lines (Boltz et al 1979; Essock 1990; Vogels and Orban 1986).

Currently three different, psychological mechanisms are considered as underlying orientation-invariant shape recognition (Cutzu and Edelman 1994; Edelman and Bülthoff 1992; Jolicoeur 1991; Tarr and Pinker 1989). One mechanism is supposed to rely on a view-independent object representation that is devoid of any particular orientation. It is assumed to be based on propositional feature-list principles and to deal with the orientation invariance of stimuli that are well discriminable and do not yield a rotation effect. Clearly, our basically easy-to-discriminate polygons and lines fall under this category. Another mechanism is supposed to involve a canonically oriented shape representation and a time-consuming rotation process to correct for aberrant orientations. It is said to be based on analogical, imagery-based principles and seems to concern stimuli that are difficult to discriminate. We assume that our basically difficultto-discriminate polygons (mirror-image and non-mirror-image) belong to this category. The third mechanism is thought to involve a multiple-orientation representation of an object, thus requiring only minor interpolative rotations to achieve invariance. It is supposed to deal with stimuli that are well familiar to the observer. This latter mechanism is presumably involved in the already-mentioned widened uprightness phenomenon (Young et al 1980). However, we would plead that none of these mechanisms should be envisaged as being neatly distinct. Rather, as we have shown with respect to at least the first two, they may function in an interactive fashion. Also, we would suggest that the analogical image metaphor connected with the second mechanism may be rather misleading. We believe that we have made it plausible that the rotation effect demonstrated with complex shapes could be based on the rotation of shape elements. However, we also doubt whether the alternative appeal to propositional operations connected with the first mechanism is really warranted. Somehow both the analogical and the propositional metaphors seem too much at odds with what is known about the neural processing in the visual system.

Acknowledgements. The research was supported by grants provided by the Deutsche Forschungsgemeinschaft. We thank Gitta Wörtwein, Chris Daneffel, Uta Werner, Ulrike Köppl, Susanne Jaeger, and Robert Bonin for varied assistance. We are grateful to Professor Rolf Ulrich and Andy Wohlschläger for comments on an earlier draft. We also tha $k$ Caroline Roberts for improving the English.

References

Attneave F, 1956 "The quantitative study of shape and pattern perception" Psychological Bulletin $53452-471$

Aulhorn O, 1948 "Die Lesegeschwindigkeit als Funktion von Buchstaben- und Zeilenlage" (The reading speed as a function of letter and row orientation) Pflügers Archiv für die Gesamte Physiologie der Menschen und der Tiere 250 12-25

Bethell-Fox C E, Shepard R N, 1988 "Mental rotation: Effects of stimulus complexity and familiarity" Journal of Experimental Psychology: Human Perception and Performance 14 12-23

Boltz R L, Harweth R S, Smith E L, 1979 "Orientation anisotropy of visual stimuli in rhesus monkey: A behavioral study" Science $205511-513$

Bornstein M H, Gross C G, Wolf J Z, 1978 "Perceptual similarity of mirror images in infancy" Cognition $689-116$

Bradshaw J L, Bradley D, Patterson K, 1976 "The perception and identification of mirrorreversed patterns" Quarterly Joumal of Experimental Psychology $28221-246$

Bradshaw J L, Nathan G, Nettelton N C, Wilson L, Pierson J, 1987 "Why is there a left under" estimation in rod bisection?" Neuropsychology $25735-738$ 
Cooper L A, 1975 "Mental rotation of random two-dimensional shapes" Cognitive Psychology 7 $20 \cdots 43$

Cooper L A, Podgorny P, 1976 "Mental transformations and visual comparison processes" Journal of Experimental Psychology: Human Perception and Performance 2 503-514

Corballis M C, 1988 "Recognition of disoriented shapes" Psychological Review $95115-123$

Corballis M C, Beale I L, 1976 The Psychology of Left and Right (Hillsdale, NJ: Lawrence Erlbaum Associates)

Corballis M C, Nagourney B A, 1978 "Latency to categorise alphanumeric characters as letters or digits" Canadian Journal of Psychology 32 186-188

Corballis M C, Zbrodoff N J, Shetzer L, Butler P B, 1978 "Decisions about identity and orientation of rotated letters and digits" Memory and Cognition $698-107$

Cutzu F, Edelman S, 1994 "Canonical views in object representation and recognition" Vision Research $343037-3056$

Delius J D, Hollard V D, 1987 "Orientation invariance of shape recognition in forebrain-lesioned pigeons" Behavioral Brain Research $23251-259$

Delius J D, Hollard V D, 1995 "Orientation invariance in pattern recognition by pigeons and humans" Journal of Comparative Psychology $109278-290$

Edelman S, Bülthoff $\mathrm{H} \mathrm{H}, 1992$ "Orientation dependence in recognition of familiar and novel views of three-dimensional objects" Vision Research $122385-2400$

Eley M G, 1983 "Symbol complexity and symbol identification with rotated symbols" Acta Psychologica $5327-35$

Essock E A, 1990 "The influence of stimulus length on the oblique effect of contrast sensitivity" Vision Research $301243-1246$

Folk M D, Luce R D, 1987 "Effects of stimulus complexity on mental rotation rate of polygons" Journal of Experimental Psychology: Human Perception and Performance $13395-404$

Foster D H, Mason R J, 1979 "Transformation and relational-structure schemes for visual pattern recognition" Biological Cybernetics $3285-93$

Gellermann $\mathrm{C} \mathrm{W}, 1933$ "Chance orders of alternating stimuli in visual discrimination experiments Journal of Genetic Psychology 42 206-208

Goebel R P, 1990 "The mathematics of mental rotations" Journal of Mathematical Psychology 34 $435-440$

Georgopoulos A P, Lurito J T, Petrides M, Schwartz A B, Massey J T, 1989 "Mental rotation of the neuronal population vector" Science $234234-236$

Hartley A A, 1981 "Mental measurement of line length: the role of the standard" Journal of Experimental Psychology: Human Perception and Performance $7309-317$

Hock H S, Tromley C L, 1978 "Mental rotation and perceptual uprightness" Perception \& Psychophysics $24529-533$

Hollard V D, Delius J D, 1982 "Rotational invariance in yisual pattern recognition by pigeons and humans" Science 218 804-806

Ittelson W H, Mowafy L, Magid D, 1991 "The perception of mirror-reflected objects" Perception $20567-584$

Jolicoeur P, 1985 "The time to name disoriented natural objects" Memory and Cognition $13289-303$

Jolicoeur P, 1991 "Identification of disorientated objects: a dual-systems theory", in Understanding Vision, An Interdisciplinary Perspective Ed.G W Humphreys (Basil Blackwell: Oxford) pp $180-198$

Jolicoeur P, Landau M J, 1984 "Effects of orientation on the identification of simple visual patterns" Canadian Journal of Psychology 38 80-93

Kolers P A, Perkins D N, 1969 "Orientation of letters and errors in their recognition" Perception \& Psychophysics $5265-269$

Koriat A, Norman J, 1985 "Mental rotation and visual familiarity" Perception \& Psychophysics $37429-439$

Kossiyn S M, Pinker S, Smith G E, Schwartz S P, 1979 "On the demystification of mental imagery" Behavioural and Brain Sciences $2535-581$

Larsen A, 1985 "Pattern matching: effects of size ratio, angular difference in orientation and familarity" Perception \& Psychophysics 38 63-68

Lohmann A, Delius J D, Hollard V D, Friesel M, 1988 "Discrimination of shape reflections and shape orientations by Columba livia" Journal of Comparative Psychology $1023-13$

Masin S C, Agostini A, 1991 "Attentional scanning and space errors" Perception \& Psychophysics 50 $285-289$

Messner R, Szu H, 1985 "An image processing architecture for real time generation of scale and rotation invariant patterns Computer Vision, Graphics and lmage Processing 31 50-66 
Milner A D, Brechman M, Pagliarini L, 1992 "To halve and to halve not: an analysis of line bisection judgements in normal subjects" Neuropsychologia $30515-526$

Morgan M J, 1983 "Mental rotation: a compositionally plausible account of transformation through intermediate steps" Perception 12 203-211

Ono H, 1967 "Difference thresholds for stimulus length under simultaneous and nonsimultaneous viewing conditions" Perception \& Psychophysics $201-207$

Pellizer G, Georgopoulos A P, 1993 "Common processing constraints for visuomotor and visual mental rotation" Experimental Brain Research $93165-172$

Perret D I, Smith P A J, Potter D D, Mistlin A J, Head A S, Milner A D, Jeeves M A, 1984 "Neuron responses to faces in the temporal cortex: studies of functional organization, sensitivity to identity and relation to perception" Human Neurobiology 3197-208

Petrusic W M, Varro L, Jamieson D G, 1978 "Mental rotation validation of two spatial ability tests" Psychologial Research $\mathbf{4 0 1 3 9 - 1 4 8}$

Pierret A, Peronnet F, 1994 "Mental rotation and mirror-image discrimination" Perceptual and Motor Skills $78515-524$

Pylyshyn Z,W, 1979 "The rate of 'mental rotation' of images: a test of a holistic analogue hypothesis" Memory and Cognition 7 19-28

Pylyshyn Z W, 1981 "The imagery debate: analogue media versus tacit knowledge" Psychological Review 88 16-45

Reed S K, Hock H S, Lockhead G R, 1993 "Tacit knowledge and the effect of pattern configuration on mental scanning" Memory and Cognition 11 137-143

Reitboek H J, Altman J, 1984 "A model for size- and rotation-invariant pattern processing in the visual system" Biological Cybernetics $51113-121$

Simion F, Bagnara S, Roncato S, Umilta C, 1982 "Transformation processes upon the visual code" Perception \& Psychophysics 31 13-25

Sheng Y, 1989 "Fourier - Mellin spatial filters for invariant pattern recognition" Optical Engineering $28494-500$

Shepard R N, Cooper L A, 1982 Mental Images and Their Transformations (Cambridge, MA: MIT Press)

Shepard R N, Metzler J, 1971 "Mental rotation of three-dimensional objects" Science $171701-703$

Siegel S, Castellan N J, 1988 Nonparametric Statistics for the Behavioral Sciences (New York: McGraw-Hill)

Takano Y, 1989 "Perception of rotated forms: a theory of information types" Cognitive Psycholog. $211-59$

Tarr M J, Pinker S, 1989 "Mental rotation and orientation-dependence in shape recognition" Cognitive Psychology $21233-282$

Tilgner R D, Hauske G, 1980 "Untersuchung zur Rotationsinvarianz bei der Erkennung von Buchstaben" (Investigation into the rotation invariance of the recognition of letters) Zeitschrift für Angewandte und Experimentelle Psychologie 27 147-162

Turnball O H, McCarthy $\mathrm{R}$ A, 1996 "Failure to discriminate between mirror-image objects: a case of viewpoint-independent object recognition?" Neurocase 2 63-72

Ulrich R, Miller J, 1994 "Effects of truncation on reaction time analysis" Journal of Experimental Psychology, General $12334-80$

Valentine T, Bruce V, 1988 "Mental rotation of faces" Memory and Cognition 16 556-566

Vauclair J, Fagot J, Hopkins W D, 1993 "Rotation of mental images in baboons when the visual input is directed to the left cerebral hemisphere" Psychological Science 499-103

Verillo R T, Irvin G, 1979 "Absolute estimation of the length as a function of orientation and contrast polarity" Sensory Processes $3261-274$

Vogels R, Orban G A, 1986 "Decision processes in visual discrimination of line orientation" Journal of Experimental Psychology: Human Perception and Performance $12115-132$

White $\mathrm{M} \mathrm{J}, 1980$ "Naming and categorization of tilted alphanumeric characters do not require mental rotation" Bulletin of the Psychonomic Society $15153-156$

Young J M, Palef S R, Logan G D, 1980 "The role of mental rotation in letter processing by children and adults" Canadian Journal of Psychology 34265 -259

Zimmer H, Fröhlich W D, 1991 "Merkmalsidentifikation und 'Mentale Rotation' unter Automatisierungsbedingungen" (Feature identification and mental rotation under automated conditions) Zeitschrift für Angewandte und Experimentelle Psychologie 38 149-167 